\title{
Analysis of Genomic DNA Methylation and Gene Transcription Modulation Induced by DNMT3A Deficiency in HEK293 Cells
}

\author{
Mengxiao Zhang \\ Shanghai Jiao Tong University \\ Jiaxian Wang \\ VU University Medical Center
}

Qiuxiang Tian

Jilin University

Lei Feng

Shanghai Jiao Tong University

Hui Yang

Shanghai Jiao Tong University

Nan Zhu

Shanghai Jiao Tong University

Xingchen Pan

Jilin University

Jianwei Zhu

Shanghai Jiao Tong University Affiliated Chest Hospital

\section{Peng Chen}

Jilin University

Huili Lu ( $\square$ roadeer@sjtu.edu.cn )

Shanghai Jiao Tong University https://orcid.org/0000-0002-8748-7899

\section{Research}

Keywords: DNMT3A, deficiency, CRISPR/Cas9, RNA-seq, methylation

Posted Date: August 17th, 2020

DOI: https://doi.org/10.21203/rs.3.rs-53272/v1

License: (c) (1) This work is licensed under a Creative Commons Attribution 4.0 International License.

Read Full License 


\section{Abstract}

Background DNA methylation is an important epigenetic modification associated with transcriptional repression, and plays key roles in normal cell growth as well as oncogenesis. Among the three main DNA methyltransferases (DNMT1, DNMT3A, and DNMT3B), DNMT3A mediates de novo DNA methylation with partial functional redundancy with DNMT3B. However, the general effects of DNMT3A and its downstream gene regulation profile are yet to be unveiled.

Results In the present study, we used CRISPR/Cas9 technology to successfully create DNMT3A deficient human embryonic kidney cell line HEK293, with frameshift mutations in its catalytic domain. Our results showed that the cell growth slowed down in DNMT3A knockout cells. UPLC-MS analysis of DNMT3A deficient cells showed that the genome-level DNA methylation was reduced by $21.5 \%$ and led to an impairment of cell proliferation as well as a blockage of MAPK and PI3K-Akt pathways. Whole genome RNA-seq revealed that DNMT3A knockout up-regulated expression of genes and pathways related to cell metabolism but down-regulated those involved in ribosome function, which explained the inhibition of cell growth and related signal pathways. Further, bisulfite DNA treatment showed that DNMT3A ablation reduced the methylation level of $D N M T 3 B$ gene as well, indicating the higher DNMT3B activity and thereby explaining those down-regulated profiles of genes.

Conclusions Our work is the first report on the effect of DNMT3A disruption in its catalytic domain, demonstrating that DNMT3A plays a key role on genomic DNA methylation and expression, and suggesting that DNMT3A could be an ideal target for the development of personalized treatment or to predict tumor prognosis.

\section{Introduction}

DNA methylation is an epigenetic modification with widespread effects on gene expression. High levels of promoter DNA methylation is usually associated with gene silencing [1]. Abnormal DNA methylation is involved in the development of multiple malignancies, such as solid tumors and leukemia [2-8]. In vertebrates, cytosine methylation on $\mathrm{CpG}$ dinucleotides is the predominant form of methylation catalyzed by DNA methyltransferase 1 (DNMT1) [9] and established de novo by DNMT3A and DNMT3B [10, 11].

To investigate the underlying mechanisms responsible for locus-specific or global methylation, in vivo and in vitro models of DNMTs deficiency have been developed [12]. In mice, knockout of DNMT1 or DNMT3B can cause early embryo death. In contrast, DNMT3A knockout mice can be born normally but develop developmental defects and die premature soon after birth [10]. These observations highlight that DNMT3A plays specific roles in regulating chromatin methylation during the development after birth [10, 13]. Similarly, in human embryonic cells, individual or simultaneous disruption of DNMT3A or DNMT3B resulted in viable, pluripotent cell lines, but deletion of DNMT1 resulted in rapid cell death [14]. Banaszak et al. mutated DNMT3A in K562 leukemia cells and the derived cell lines showed impaired cell growth [2]. Although almost all cells can survive DNMT3A mutation, reports have shown paradoxical hyper- 
methylation of genes, or no changes in global or regional DNA methylation patterns in response to DNMT3A knockdown $[2,15]$. Hence, the exact roles of DNMT3A are yet to be elucidated.

CRISPR/Cas9 system is an efficient genome editing technique developed in recent years [16]. Comparing with the traditional knockout techniques, such as zinc finger nuclease technology (ZFN) and transcriptional activation effect factor nuclease technology (TALEN), CRISPR/Cas 9 is comparatively easy to implement, is cost and time-effective, as well as has higher efficiency. CRISPR/Cas9 technique has been successfully used in human cells, zebrafish, mice, and bacterial genome modification $[17,18]$. In the present study, we used CRISPR/Cas9 technology to establish a DNMT3A knockout cell line derived from HEK293T, a human embryonic kidney cell line. We performed detailed transcriptomic and epigenetic analyses, in addition to physiology measurements, to discover the impact of DNMT3A deficiency on cell proliferation and metabolism, as well as to identify genes which are potentially regulated by DNMT3A.

\section{Materials And Methods}

\section{Cell culture and reagents}

Wild type HEK293 cells (HEK293T) were obtained from the Type Culture Collection of the Chinese Academy of Sciences (Shanghai, China) and detected to be negative for mycoplasma contamination using the Myco-Blue mycoplasma detector (Vazyme; Nanjing, Jiangsu, China). Cells were cultured in high glucose DMEM supplemented with $10 \% \mathrm{FBS}$, incubated at $37^{\circ} \mathrm{C}$ with $5 \% \mathrm{CO}_{2}$ in a humidified cell incubator (Thermo Fisher Scientific; OH, USA). The plasmid pX330 carrying CRISPR/Cas9 system was kindly provided by Dr. Feng Zhang (MIT) [19]. Competent cells of the E. coli strains DH5a were purchased from Microgene (Shanghai, China). All media and supplements were purchased from Gibco (Thermo Fisher Scientific; Waltham, MA, USA). Cell growth and viability were monitored with a cell counter (Countstar; Shanghai, China).

\section{SgRNA design and DNMT3A disruptive vector construction}

Two sgRNAs targeting exon 19 of DNMT3A (GeneBank ID 806904736) were designed using the web tool provided by Dr. Zhang's lab (http://crispr.mit.edu) as shown in Fig. 1. To construct the sgRNA plasmids, single strand primers were designed and synthesized as sgRNA1-forward: 5'CACCGCATGATGCGCGGCCCAAGG-3', sgRNA1-reverse 5'-AAACCCTTGGGCCGCGCATCATGC-3', sgRNA2forward 5'-CACCGCTCACTAATGGCTTCTACCT-3' and sgRNA1-reverse 5'-

AAACAGGTAGAAGCCATTAGTGAGC-3'. Each pair of primers were annealed to generate double-stranded cDNA, phosphorylated by T4 polynucleotide kinase at the $5^{\prime}$ ends (NEB, Ipswich, MA) at $37^{\circ} \mathrm{C}$ for 30 min, and further ligated into Bbsl digested pX330 plasmids by T4 DNA ligase (Takara; Kusatsu, Shiga, Japan). The ligate was transformed to DH5a competent cells for culture overnight. Then the grown clones were selected for sequencing to get the right constructed plasmids pX330-sgRNA1 and pX330-sgRNA2.

\section{Transfection of HEK293 cells}


HEK293 cells were seeded at $2 \times 10^{5}$ cells/well into 12-well plate one day prior to transfection. When reached $70-80 \%$ confluence, the cells were co-transfected with pX330-sgRNA1 and pX330-sgRNA2 at a molar ratio of 1:1, since it was reported that double sgRNAs could result in higher editing efficiency than single one [20]. The transfection was performed using Lipofectamine 2000 reagent (Invitrogen, CA, USA) according to manufacturer's instructions.

\section{DNMT3A knockout clones selection}

HEK293 cell pool transfected with pX330-sgRNAs were seeded into 96-well plates at the density of 0.5 cell per well for limiting dilution. After about ten days' incubation, the plates were examined for single cell clones under microscope. When grew to about $80 \%$ confluent in the well, the clones would be detached for subpopulation and the genomic DNA was extracted with QuickExtract DNA extraction solution (Epicenter; MD, USA) for PCR verification, using primers HEK293-DNMT3A-For (5'GTACCATCCTGTCCCCTCCAC-3') and HEK293-DNMT3A-Rev (5'-GGCTCAGGGTTAAACGGGGA-3'), which can amplify a 798 bp fragment for HEK293 wild-type cells. By sequencing the amplified fragments, the clone with disrupted DNMT3A was selected and designated to be DNMT3A KO cell line.

\section{DNMT3A knockout cells proliferation curve}

DNMT3A KO and WT cells were cultured and seeded at $3 \times 10^{4}$ cells/well into 12-well plate. Cells were counted every $24 \mathrm{~h}$ for consecutive 6 days. And cell proliferation curves were compared between the two cell lines.

\section{Western blot analysis}

DNMT3A KO and WT HEK293 cells of $1 \times 10^{6}$ were washed with PBS, lysed using $100 \mu \mathrm{L}$ RAPA lysis buffer containing protease inhibitors cocktail (Roche; Penzberg, Germany), and separated by a $10 \%$ SDSPAGE. After transferring onto a $0.45 \mu \mathrm{m}$ PVDF membranes, immunoblotting was performed. For detection of DNMT3A deficiency, primary mouse monoclonal antibody against GAPDH (Sangon; Shanghai, China) and polyclonal rabbit-anti-human DNMT3A (Sangon) were used at 1:1000 dilution. For detection of MAPK and PI3K-Akt pathways, primary monoclonal antibodies against human Erk (137F5; Cell Signaling Technology; Danvers, MA, USA), phosphor-Erk (197G2; Cell Signaling Technology), JNK (D-2; Santa Cruz; Dallas, TX, USA), phosphor-JNK (G9; Cell Signaling Technology), Akt (11E7; Cell Signaling Technology), and phosphor-Akt (244F9; Cell Signaling Technology) were used. HRP-conjugated anti-mouse IgG or antirabbit IgG antibodies (Jackson ImmunoResearch; PA, USA) were used for secondary antibodies. Signals were detected with enhanced chemiluminescence (Millipore; MA, USA) and visualized with a gel imaging system (Tanon; Shanghai, China).

\section{Genome-wide DNA methylation analysis by UPLC-ESI-MS/MS}

Genomic DNA of cells were extracted by AxyPrep Kit (Axygen; Hangzhou, Zhejiang, China) and RNase A was added to remove RNA. Then the genomic DNA was hydrolyzed by DNase I at $37^{\circ} \mathrm{C}$ for $1 \mathrm{~h}$, denatured 
at $100^{\circ} \mathrm{C}$ for $3 \mathrm{~min}$, and immediately cooled down on ice for $10 \mathrm{~min}$, then treated with Nuclease P1 at $37^{\circ} \mathrm{C}$ for $16 \mathrm{~h}$, followed by treatment of alkaline phosphatase at $37^{\circ} \mathrm{C}$ for $2 \mathrm{~h}$. The nucleotides were stored at $-20^{\circ} \mathrm{C}$ before UPLC-ESI-MS-MS detection.

Acquity UPLC (Waters $₫$ USA) coupled with Triple Quad ${ }^{\text {TM }} 5500$ mass spectrometry (Sciex冈USA) was used to quantitatively analyze $\mathrm{m}^{5} \mathrm{dC}$ and $\mathrm{dG}$. UPLC-ESI-MS/MS method was established to evaluate DNA methylation status of genome [21]. Reference nucleotide standards of $A, G, T, C, d A, d G, d C, U$ and $m^{5} d C$ were purchased from Sigma (Sigma Aldrich, St. Louis, MO, USA) and dissolved in $\mathrm{H}_{2} \mathrm{O}$ to a final concentration of $1.0 \mathrm{mg} / \mathrm{ml}$. UPLC and electronic spray were used to separate and detect the standards at multiple reaction monitoring (MRM) mode. The $\mathrm{m}^{5} \mathrm{dC} \triangle \mathrm{m} / \mathrm{z} 241.9 \rightarrow 126.3 \rrbracket a n d \mathrm{dG}(\mathrm{m} / \mathrm{z} 268.1 \rightarrow 152.3)$ were chosen as parent and child ion pairs for quantitative detection. The CE voltage of both $\mathrm{m}^{5} \mathrm{dC}$ and $\mathrm{dG}$ was $15 \mathrm{eV}$, and the DP voltage was $40 \mathrm{~V}$, respectively. Standard curves of $\mathrm{m}^{5} \mathrm{dC}$ and dG were first graphed and the level of cytosine methylation was calculated as $\left(\mathrm{m}^{5} \mathrm{dC} / \mathrm{dG}\right) \times 100 \%$.

\section{RNA-seq to reveal transcriptional response to DNMT3A deficiency}

Total RNA was extracted from $10^{6}$ of DNMT3A KO or WT cells. Oligo(dT) magnetic beads were used to enrich mRNA. CDNA was obtained using Illumina Truseq ${ }^{\text {TM }}$ RNA sample prep Kit, and pair-end sequencing (insert size $=300 \mathrm{bp}$, read length $=150 \mathrm{bp}$ ) was performed according to the standard protocol of Novaseq 6000 (Illumina, CA, USA). Raw sequencing reads were filtered to include only high quality reads in downstream analysis: 1) clip adapter sequence from reads, and remove reads with no insertion; 2) clip 3' low quality bases (Phred quality < 20), and remove the whole read if there exists a single base with Phred quality < 10;3) remove the reads that have more than $10 \%$ ambiguous bases $(\mathrm{N})$; remove the reads that are shorter than $20 \mathrm{bp}$ after clipping. The filtered reads were aligned to human transcriptome (build GRCh38) by TopHat [22]. PCR duplicates were marked and ignored in downstream analysis. All the data were deposited into the open-access Genome Sequence Archive (gsa.big.ac.cn) under accession no. CRA002294.

The read count data of DNMT3A KO and WT cells was analyzed by Cufflink software to identify the differential gene expression induced by DNMT3A deficiency [23]. We used FPKM (Fragments Per Kilobase of exon model per Million mapped reads) to estimate genes expression levels. False discovery rate (FDR) p values were calculated using the method proposed by Benjamini and Hochberg (1995) to correct for multiple testing. Differentially expressed genes in DNMT3A KO cells were identified by FDR $p$ value $\leq$ 0.05 and absolute logarithm of fold change $\left(\log _{2} F C\right) \geq 2$.

\section{KEGG pathway analysis of differentially expressed genes}

For the purpose of pathway enrichment analysis, we defined differential expression using a loose definition (FDR p value $\leq 0.05$ and absolute $\log _{2} F C \geq 1$ ). The Ensembl IDs of differentially expressed genes were analyzed by KOBAS (http://kobas.cbi.pku.edu.cn) for KEGG pathway enrichment. The pathways with FDR $p$ value $\leq 0.05$ were considered significantly differentially expressed. 


\section{Bisulfite DNA analysis and quantitative PCR verification of DNMT3A regulated genes}

DNMT3A is responsible for the de novo methylation of multiple genes, and its mutation can lead to demethylation of promoter $\mathrm{CpG}$ and thus elevate gene expression at the transcript level, which further upregulate or down-regulate related downstream genes indirectly. Therefore, from the gene pool which transcript level was interfered by DNMT3A knockout as determined by RNA-seq, we selected 3 representative genes to verify by bisulfite DNA analysis as well as quantitative PCR: RUNX1, IQGAP3, and DNMT3B. RUNX1 is known to be regulated by DNMT3A in hematopoietic carcinogenesis [24]. IQGAP3 is a scaffolding protein that is involved in cancer cells proliferation, and with no correlation with DNA methyltransferases reported before [25]. All 3 genes were hot studied in malignancy development and helpful to understand the functions of DNMT3A.

DNA methylation status of selected genes were analyzed by bisulfite sequencing PCR (BSP). Genomic DNA was extracted with an Axygen Genomic DNA Miniprep Kit (San Francisco, CA, USA), and $0.5 \mu \mathrm{g}$ of DNA was modified through bisulfite treatment using a Bisuldream ${ }^{\circledR}$ - Methylation Universal kit (Miozyme; Shanghai, China). Bisulfite-PCR of the genes promoter regions (Table S1) was performed using the following specific primers: RUNX1 forward: 5'- TTTTTAGGTTTTAAAATATTTGTGAGTTGT-3', RUNX1 reverse: 5'- CACCTACCCTCCCCCAAACTATAC-3', IAGAP3 forward: 5'-

GTAGAAAAGGAGTTTGGAAGGAATAAGA-3', IQGAP3 reverse: 5'- ACTCACAAACTACCCAACCTAAACC-3', and DNMT3B forward 5'- TTAAAGTAGGATGATAGGTAGGGGTAT-3', DNMT3B reverse: 5'CCCTAAAAAATCAAAAACCCTAAAC-3'. The amplified fragments were inserted into pMD19-T vectors (Takara; Tokyo, Japan), and 10-15 clones for each gene were selected for sequencing. The results were analyzed by a web-based quantification tool for methylation analysis (http://quma.cdb.riken.jp).

To detect the transcription levels of the above selected three genes, the DNMT3A KO and WT HEK293 cells were cultured and RNA samples were extracted using Direct-zol RNA kit (Zymo Research; Irvine, CA, USA). Then cDNA was synthesized according to the protocol of the RT-PCR kit (Takara; Kusatsu, Shiga, Japan) and used as templates for quantitative PCR. The primers were designed using Primer Primier 5.0 (Premier Biosoft; Palo Alto, CA, USA) according to published sequences (NCBI Accession number: D43967 for RUNX1, AB105103 for IQGAP3, AF156487 for DNMT3B and M33197 for GAPDH). The following sequences for primers were synthesized (Sangon Biotech; Shanghai, China) as RUNX1 forward: $5^{\prime}$ TCTCTTCCTCTATCTTCCA- 3', RUNX1 reverse: 5'-GGTATGTGCTATCTGCTTA-3'; IQGAP3 forward: 5' GACCACTACCTAACTCAG-3', IQGAP3 reverse 5'-GCATCATCAACAACTTCTA-3'; DNMT3B forward: 5'GGCAAGTTCTCCGAGGTCTCTG-3', DNMT3B reverse: 5'-TGGTACATGGCTTTTCGATAGGA-3'; and GAPDH forward: 5'-CTCTGGTAAAGTGGATATTGT-3', GAPDH reverse: 5'- GGTGGAATCATATTGGAACA-3'). The real-time PCR procedures were performed with $25 \mu \mathrm{L}$ PCR reaction systems including $12.5 \mu \mathrm{L}$ qPCR Mix (Toyobo; Osaka, Japan), $0.4 \mu \mathrm{M}$ of each primer, and $1 \mu \mathrm{L}$ template cDNA by thermocycler (StepOnePlus; ThermoFisher, USA). The delta-delta threshold cycle $\left(\mathrm{C}_{\mathrm{T}}\right)$ method was used to calculate relative copy numbers of targeted genes related to housekeeping gene GAPDH.

\section{Results}




\section{Generation of DNMT3A deficient clones of HEK293}

Plasmids pX330-sgRNA1 and pX330-sgRNA2 were co-transfected into HEK293 cells. After limiting dilution, the grown clones were selected by PCR using the HEK293-DNMT3A-For and HEK293-DNMT3ARev verification primers. We identified one DNMT3A deficient clone from 17 clones, which showed complete disruption of DNMT3A gene and designated it as DNMT3A KO (Figure 2A). Figure 2B shows 137 and 10 bp deletions in the KO A and KO B alleles respectively, leading to complete ablation of DNMT3A due to frameshift mutations. Next, we performed western blot to characterize the expression of DNMT3A in the DNMT3A KO clone. As shown in Figure 2C, DNMT3A protein expression was completely abrogated in the selected clone, thereby confirming the successful ablation of DNMT3A.

\section{DNMT3A deficiency resulted in genome-wide decrease in DNA methylation}

DNMT3A is responsible for the DNA methylation of large number of genes in mammalian cells. To further verify the effect of DNMT3A, we performed UPLC-MS to quantify the global DNA methylation level changes following DNMT3A knockout. As described in Materials and Methods, we first characterized the peaks of standards $A, G, T, C, d A, d G, d C, U$, and $m^{5} d C$, and then developed the linear curves of $d G$ and $\mathrm{m}^{5} \mathrm{dC}$ (Figure S1). Genomic DNA were extracted from DNMT3A KO and WT cells and hydrolyzed to nucleotides for the measurement of $d G$ and $m^{5} d C$ content. The percentage of $m^{5} d C / d G$ was calculated to represent the methylation level. As shown in Figure 3, the whole-genome DNA methylation level decreased significantly (by $21.5 \%)$ in DNMT3A KO cells $(12.35 \pm 0.36 \%)$ than in WT cells $(9.69 \pm 0.13 \%)$.

\section{DNMT3A deficiency impaired cell growth}

To evaluate the effect of DNMT3A deficiency on cell proliferation, growth profiles of DNMT3A KO and WT cells were evaluated as shown in Figure 4. The proliferation ability of HEK293 cells was significantly reduced in response to DNMT3A deficiency. After 6 days, the cell counts of DNMT3A KO cells reduced to only $40 \%$ of WT cells $(0.77 \pm 0.15) \times 10^{6}$ vs $(1.94 \pm 0.17) \times 10^{6}$ cells $)$. Further, the doubling time was notably prolonged from $0.99 \pm 0.28$ days for WT cells to $1.53 \pm 0.39$ days for DNMT3A KO cells.

\section{RNA-seq analysis}

After clipping and filtering, RNA-seq yielded sequencing data of 53.2 million reads ( 7.9 billion base pairs) and 54.8 million reads ( 8.2 billion base pairs) for DNMT3A KO and WT cells, respectively. It was equivalent to 264.8 and 273.5 times coverage of human transcriptome (30 million base pairs in size). At least $98.4 \%$ of the bases had Phred quality $>20$ (error rate $<0.01 \%$ ). TopHat mapped $94.0 \%$ of sequencing reads to human genome, including $3.4 \%$ reads mapped to multiple genomic position which were excluded from the expression analysis.

\section{Differentially expressed genes and pathways}


At significant level of FDR $p$ value $\leq 0.05$ and with absolute $\log _{2} F C \geq 2$, we identified 51 differentially expressed genes (Figure 5). Among them, more genes were down-regulated $(\mathrm{N}=34)$ as compared to upregulated genes $(\mathrm{N}=17)$. The top 10 differentially methylated genes are listed in Table 1 (FDR $p$ value $\leq$ $2.46 \times 10^{-42}$ ). The pathway enrichment analysis was performed for 815 up-regulated and 658 downregulated Ensembl IDs (FDR p value $\leq 0.01$ and absolute fold change $\geq 1.5$ ). Pathways related to calcium signaling, ECM-receptor interaction, and Hippo signaling, were up-regulated (Table 2), while pathways including Ribosome biogenesis and cysteine and methionine metabolism were down-regulated (FDR p value $\leq 0.01$, Table 3 ).

\section{Methylation status and transcript level of representative genes regulated by DNMT3A deficiency}

According to the results of RNA-seq, there were many up- or down-regulated genes (Figure 5), indicating the alteration in methylation profiles caused by DNMT3A deficiency. Since both DNMT3A and DNMT3B are responsible for de novo DNA methylation together, the RNA-seq signal of DNMT3B was determined. We observed a 1.31-fold increase in the RNA-seq signal of DNMT3B in DNMT3A KO cells compared to that in WT, indicating the possible compensatory effect of DNMT3B at the deficiency of DNMT3A. Upregulation of DNMT3B may result in the methylation of some genes and the reduction of their transcription, which explains why the transcription levels of some genes were reduced in this study.

The promoter methylation levels and mRNA transcription levels of $D N M T 3 B$ and two representative tumorigenesis-related genes, RUNX1 and IQGAP3, were verified. The $C p G$ island-rich promoter region (from -1.0 to $0 \mathrm{~kb}$ relative to the transcription start site) was analyzed by BSP for each of the three genes. According to the results shown in Figure 6, DNMT3A deficiency did reduce the DNA methylation level of RUNX1 promoter (Figure 6A), but it induced the methylation of IQGAP3 promoter (Figure 6B); this induction of methylation was possibly caused by $D N M T 3 B$, which showed reduced DNA methylation in its promoter region (Figure $6 \mathrm{C}$ ). Quantitative PCR results confirmed the methylation regulation results. Transcription of $R U N X 1$ was up-regulated by $80 \%$, and that of IQGAP3 was reduced by $46 \%$. The transcription of $D N M T 3 B$ was also elevated in DNMT3A KO cells, but only by $15 \%$ (Figure 6D). This is the first report to show that DNMT3A contributes to the methylation of the DNMT3B gene, indicating the cross-activity of the two de novo DNA methyltransferases.

\section{Discussion}

In recent years, DNMT3A has been intensely studied for its role in tumor prognosis or therapy $[3,26]$. To better reveal the functions of DNMT3A in cancer occurrence and development, in this study, we mutated HEK293 cells using the CRISPR/Cas9 technology and successfully created a DNMT3A knockout cell line, with homozygous frameshift deletion in both alleles. LC-MS analysis showed that knockout of DNMT3A induced a $21.5 \%$ reduction of global DNA methylation. The reserved DNA methylation could be attributed to the functions of DNMT1 and DNMT3B [14]. In addition, we attempted the mutation of DNMT1 or DNMT3B using the same strategy in HEK293 cells, but no single clone with the required gene mutation or deficiency was accessed, or the selected clones were unstable for long-term culture (data not shown). 
Several previous studies have focused on DNMT3A gene knockout in human or mouse-resourced cells, including human embryotic stem cells, human leukemia cells K562, mouse hematopoietic stem cells, as well as mouse somatic cells $[2,14,27,28]$. Compared to mouse cells, human cells are less tolerant to DNMT3A deficiency and it can cause lethality and genomic instability in the cells. The results of these previous studies were consistent with our observations that DNMT3A deficiency suppresses HEK293 cell activity. The doubling time of cells dropped from $0.99 \pm 0.28$ days to $1.53 \pm 0.39$ days; this result was similar to the phenomenon of impaired cell growth caused by the DNMT3A mutation in K562 cells [2]. We assume that this effect is associated with the MAPK or PI3K-Akt pathways, which predominantly contribute to cell proliferation and migration. In this study, we also observed inhibition of the Erk, JNK, and Akt signaling pathways (Fig. 7).

A previous study introduced frameshift mutations at exons 2 and 3, ablating DNMT3A from more upstream regulatory region [2]. However, in our study, the DNMT3A mutation was targeted at exon 19 in the catalytic domain. Reduced genome-wide DNA methylation level in DNMT3A KO cells was expected to result in higher transcription levels. However, we unexpectedly observed that a high number of genes were down-regulated in our significant differential expression spectrum with FDR $p$ value $\leq 0.05$ and fold change $\geq 2$ (binomial $p$ value $=7.6 \times 10^{-3}$ ). We also found that the top 10 genes in the most significant gene cluster were down-regulated. DNMT3B showed abnormal up-regulation upon DNMT3A deficiency (Fig. 6), and possibly had a methylation function on some of the genes. However, a new research indicates that two SU(VAR)3-9 homologs, the transcriptional anti-silencing factor SUVH1, and SUVH3, as the methyl reader candidates, are associated with euchromatic methylation in vivo [29]. In plant, yeast, and mammalian cells, ectopic recruitment of DNAJ1 was shown to enhance gene transcription [30]. Therefore, the SUVH proteins bind to methylated DNA and recruit DNAJ proteins to enhance proximal gene expression, counteracting the repressive effects of transposon insertion near genes [42]. The top 10 differently expressed genes were likely associated with the SUVH1 and SUVH3 factors when methylation was decreased. However, the real reason for the down-regulation of the top 10 genes in this study is still unknown.

Further investigation of the regulated pathways helped us in understanding that the lower growth rate is a consequence of DNMT3A deficiency. The calcium signaling pathway and ECM-receptor interaction, which are genetically associated with the progression and recurrence of atrial fibrillation [31], and the Hippo signaling pathway were up-regulated in DNMT3A KO cells (FDR p values were $0.002449,0.004114$, and 0.03080 , respectively). Twist2 is known to regulate ITGA6 and CD44 expression in the ECM-receptor interaction pathway to promote kidney cancer cell proliferation and invasion [32]. The major functions of the Hippo pathway are the restriction of tissue growth in adults and modulation of cell proliferation, differentiation, and migration in developing organs [33]. Cysteine and methionine metabolism are strictly indispensable to the proliferation of porcine adipogenic precursor cells. After commitment, Met deficiency in media has also been shown to affect the differentiation into adipocytes and alter lipid accumulation [34]. 


\section{Conclusions}

In recent years, DNMT3A has been identified to be an ideal target for the development of personalized treatment or predict tumor prognosis [26]. This is the first report on the effect of DNMT3A disruption in its catalytic domain on genomic DNA methylation and expression. The genes revealed by RNA-seq to be tightly regulated by DNMT3A in HEK293 cells, in this study, are of great significance to understand the functions of DNMT3A in the origin and development of tumors, and are potential novel targets for future cancer therapy.

\section{Declarations}

\section{Ethics approval and consent to participate}

Not applicable.

\section{Consent for publication}

Not applicable.

\section{Availability of data and material}

RNA-seq data are available in the open-access Genome Sequence Archive (gsa.big.ac.cn) under accession no. CRA002294.

\section{Competing interests}

The authors declare that they have no conflict of interest.

\section{Funding}

This work was funded by the Science and Technology Commission of Shanghai Municipality (No. 17431904500 to $\mathrm{Lu} \mathrm{H})$.

\section{Authors' contributions}

Zhang M, Wang J, Tian Q, Feng L \& Yang H: Investigation, Methodology, and Data Curation; Zhang M, Wang J, Zhu N, Pan X: Investigation and Software; Zhu J, Chen P \& Lu H: Writing; Chen P \& Lu H: Supervision.

\section{Acknowledgements}

Not applicable.

\section{References}


1. You JS, Jones PA: Cancer genetics and epigenetics: two sides of the same coin? Cancer cell 2012, 22(1):9-20.

2. Banaszak LG, Giudice V, Zhao X, Wu Z, Gao S, Hosokawa K, Keyvanfar K, Townsley DM, GutierrezRodrigues F, Fernandez Ibanez MDP et al: Abnormal RNA splicing and genomic instability after induction of DNMT3A mutations by CRISPR/Cas9 gene editing. Blood cells, molecules \& diseases 2018, 69:10-22.

3. Yang SM, Huang CY, Shiue HS, Pu YS, Hsieh YH, Chen WJ, Lin YC, Hsueh YM: Combined effects of DNA methyltransferase 1 and $3 A$ polymorphisms and urinary total arsenic levels on the risk for clear cell renal cell carcinoma. Toxicology and applied pharmacology 2016, 305:103-110.

4. Montgomery KG, Liu MC, Eccles DM, Campbell IG: The DNMT3B C $\rightarrow$ T promoter polymorphism and risk of breast cancer in a British population: a case-control study. Breast cancer research : BCR 2004, 6(4):R390-394.

5. Lee SJ, Jeon HS, Jang JS, Park SH, Lee GY, Lee BH, Kim CH, Kang YM, Lee WK, Kam S et al: DNMT3B polymorphisms and risk of primary lung cancer. Carcinogenesis 2005, 26(2):403-409.

6. Fan H, Liu DS, Qiu XM, Qiao FC, Wu QX, Su XW, Zhang F, Song YW, Zhao ZJ, Xie W: A functional polymorphism in the DNA methyltransferase-3A promoter modifies the susceptibility in gastric cancer but not in esophageal carcinoma. Bmc Med 2010, 8.

7. Gao J, Wang LH, Xu JK, Zheng JM, Man XH, Wu HY, Jin J, Wang KX, Xiao HS, Li SD et al: Aberrant DNA methyltransferase expression in pancreatic ductal adenocarcinoma development and progression. J Exp Clin Canc Res 2013, 32.

8. Mirza S, Sharma G, Parshad R, Gupta SD, Pandya P, Ralhan R: Expression of DNA Methyltransferases in Breast Cancer Patients and to Analyze the Effect of Natural Compounds on DNA Methyltransferases and Associated Proteins. J Breast Cancer 2013, 16(1):23-31.

9. Bestor TH: Activation of mammalian DNA methyltransferase by cleavage of a $\mathrm{Zn}$ binding regulatory domain. The EMBO journal 1992, 11(7):2611-2617.

10. Okano M, Bell DW, Haber DA, Li E: DNA methyltransferases Dnmt3a and Dnmt3b are essential for de novo methylation and mammalian development. Cell 1999, 99(3):247-257.

11. Okano $\mathrm{M}$, Xie S, Li E: Cloning and characterization of a family of novel mammalian DNA (cytosine-5) methyltransferases. Nat Genet 1998, 19(3):219-220.

12. Huang YH, Su J, Lei Y, Brunetti L, Gundry MC, Zhang X, Jeong M, Li W, Goodell MA: DNA epigenome editing using CRISPR-Cas SunTag-directed DNMT3A. Genome biology 2017, 18(1):176.

13. Riggs AD, Xiong Z: Methylation and epigenetic fidelity. Proc Natl Acad Sci U S A 2004, 101(1):4-5.

14. Liao J, Karnik R, Gu H, Ziller MJ, Clement K, Tsankov AM, Akopian V, Gifford CA, Donaghey J, Galonska $C$ et al: Targeted disruption of DNMT1, DNMT3A and DNMT3B in human embryonic stem cells. Nat Genet 2015, 47(5):469-478.

15. Challen GA, Sun DQ, Jeong M, Luo M, Jelinek J, Berg JS, Bock C, Vasanthakumar A, Gu HC, Xi YX et al: Dnmt3a is essential for hematopoietic stem cell differentiation. Nat Genet 2012, 44(1):23-U43. 
16. Horvath P, Barrangou R: CRISPR/Cas, the immune system of bacteria and archaea. Science 2010, 327(5962):167-170.

17. Cong L, Ran FA, Cox D, Lin S, Barretto R, Habib N, Hsu PD, Wu X, Jiang W, Marraffini LA: Multiplex genome engineering using CRISPR/Cas systems. Science 2013, 339(6121):819-823.

18. Mali P, Yang L, Esvelt KM, Aach J, Guell M, DiCarlo JE, Norville JE, Church GM: RNA-guided human genome engineering via Cas9. Science 2013, 339(6121):823-826.

19. Cong L, Ran FA, Cox D, Lin SL, Barretto R, Habib N, Hsu PD, Wu XB, Jiang WY, Marraffini LA et al: Multiplex Genome Engineering Using CRISPR/Cas Systems. Science 2013, 339(6121):819-823.

20. Zheng Q, Cai X, Tan MH, Schaffert S, Arnold CP, Gong X, Chen CZ, Huang S: Precise gene deletion and replacement using the CRISPR/Cas9 system in human cells. BioTechniques 2014, 57(3):115-124.

21. Putra SED, Neuber C, Reichetzeder C, Hocher B, Kleuser B: Analysis of Genomic DNA Methylation Levels in Human Placenta using Liquid Chromatography-Electrospray lonization Tandem Mass Spectrometry. Cell Physiol Biochem 2014, 33(4):945-952.

22. Trapnell C, Pachter L, Salzberg SL: TopHat: discovering splice junctions with RNA-Seq. Bioinformatics 2009, 25(9):1105-1111.

23. Trapnell C, Roberts A, Goff L, Pertea G, Kim D, Kelley DR, Pimentel H, Salzberg SL, Rinn JL, Pachter L: Differential gene and transcript expression analysis of RNA-seq experiments with TopHat and Cufflinks. Nature protocols 2012, 7(3):562-578.

24. Stengel A, Kern W, Meggendorfer M, Nadarajah N, Perglerova K, Haferlach T, Haferlach C: Number of RUNX1 mutations, wild-type allele loss and additional mutations impact on prognosis in adult RUNX1-mutated AML. Leukemia 2018, 32(2):295-302.

25. Lin M, Liu Y, Ding X, Ke Q, Shi J, Ma Z, Gu H, Wang H, Zhang C, Yang C et al: E2F1 transactivates IQGAP3 and promotes proliferation of hepatocellular carcinoma cells through IQGAP3-mediated PKC-alpha activation. American journal of cancer research 2019, 9(2):285-299.

26. Gao XN, Yan F, Lin J, Gao L, Lu XL, Wei SC, Shen N, Pang JX, Ning QY, Komeno Y et al: AML1/ETO cooperates with HIF1alpha to promote leukemogenesis through DNMT3a transactivation. Leukemia 2015, 29(8):1730-1740.

27. Jeong M, Park HJ, Celik H, Ostrander EL, Reyes JM, Guzman A, Rodriguez B, Lei Y, Lee Y, Ding L et al: Loss of Dnmt3a Immortalizes Hematopoietic Stem Cells In Vivo. Cell reports 2018, 23(1):1-10.

28. Hatazawa Y, Ono Y, Hirose Y, Kanai S, Fujii NL, Machida S, Nishino I, Shimizu T, Okano M, Kamei Y et al: Reduced Dnmt3a increases Gdf5 expression with suppressed satellite cell differentiation and impaired skeletal muscle regeneration. FASEB journal : official publication of the Federation of American Societies for Experimental Biology 2018, 32(3):1452-1467.

29. Du J, Johnson LM, Groth M, Feng S, Hale CJ, Li S, Vashisht AA, Wohlschlegel JA, Patel DJ, Jacobsen SE: Mechanism of DNA methylation-directed histone methylation by KRYPTONITE. Molecular cell 2014, 55(3):495-504.

30. Harris CJ, Scheibe M, Wongpalee SP, Liu W, Cornett EM, Vaughan RM, Li X, Chen W, Xue Y, Zhong Z et al: A DNA methylation reader complex that enhances gene transcription. Science 2018, 
362(6419):1182-1186.

31. Buttner P, Ueberham L, Shoemaker MB, Roden DM, Dinov B, Hindricks G, Bollmann A, Husser D: Identification of Central Regulators of Calcium Signaling and ECM-Receptor Interaction Genetically Associated With the Progression and Recurrence of Atrial Fibrillation. Frontiers in genetics 2018, 9:162.

32. Zhang HJ, Tao J, Sheng L, Hu X, Rong RM, Xu M, Zhu TY: Twist2 promotes kidney cancer cell proliferation and invasion by regulating ITGA6 and CD44 expression in the ECM-receptor interaction pathway. OncoTargets and therapy 2016, 9:1801-1812.

33. Meng Z, Moroishi T, Guan KL: Mechanisms of Hippo pathway regulation. Genes \& development 2016, 30(1):1-17.

34. Castellano R, Perruchot MH, Tesseraud S, Metayer-Coustard S, Baeza E, Mercier Y, Gondret F: Methionine and cysteine deficiencies altered proliferation rate and time-course differentiation of porcine preadipose cells. Amino acids 2017, 49(2):355-366.

\section{Tables}

Table 1

Highly differentially expressed genes

\begin{tabular}{|c|c|c|c|c|c|c|}
\hline Gene ID & Gene Name & FC & $\log 2 F C$ & p-value & p-adjust & regulate \\
\hline ENSG00000128591 & FLNC & 0.0363 & -4.78 & 2.17E-175 & $4.00 \mathrm{E}-171$ & down \\
\hline ENSG00000161671 & EMC10 & 0.009 & -6.79 & $4.81 \mathrm{E}-164$ & $4.44 \mathrm{E}-160$ & down \\
\hline ENSG00000019549 & SNAI2 & 0.0632 & -3.98 & $1.35 \mathrm{E}-97$ & $8.32 \mathrm{E}-94$ & down \\
\hline ENSG00000165512 & ZNF22 & 0.0252 & -5.31 & $1.58 \mathrm{E}-95$ & 7.31E-92 & down \\
\hline ENSG00000184368 & MAP7D2 & 0.0452 & -4.47 & $1.99 \mathrm{E}-80$ & 7.35E-77 & down \\
\hline ENSG00000173530 & TNFRSF10D & 0.033 & -4.92 & $2.00 \mathrm{E}-72$ & $6.15 \mathrm{E}-69$ & down \\
\hline ENSG00000164853 & UNCX & 0.0516 & -4.27 & $9.81 \mathrm{E}-70$ & $2.58 \mathrm{E}-66$ & down \\
\hline ENSG00000121413 & ZSCAN18 & 0.0452 & -4.47 & $3.45 \mathrm{E}-51$ & $7.95 \mathrm{E}-48$ & down \\
\hline ENSG00000148798 & INA & 0.0868 & -3.53 & $1.22 \mathrm{E}-48$ & $2.51 \mathrm{E}-45$ & down \\
\hline ENSG00000131435 & PDLIM4 & 0.0543 & -4.20 & $1.34 \mathrm{E}-45$ & $2.46 \mathrm{E}-42$ & down \\
\hline \multicolumn{7}{|c|}{$\begin{array}{l}\text { Note: Gene ID: Ensembl IDs. FC: The fold change of the two samples, and wild type is the control. } \\
\text { Log2FC: The base is the base } 2 \text { logarithm of the difference between the two samples, and wild type is } \\
\text { the control. p-value: The difference in test results of the gene in two samples. p-adjust: Checked result } \\
\text { for p-value. Regulate: To indicate whether the expression of the gene is down-regulated or up- } \\
\text { regulated; "down" in the column indicates down-regulation of gene expression. }\end{array}$} \\
\hline
\end{tabular}


Table 2

Enrichment analysis of up-regulated pathways

\begin{tabular}{|c|c|c|c|c|}
\hline Database & ID & Term & p-value & p-ajusted \\
\hline KEGG Pathway & hsa05416 & Viral myocarditis & 9.17E-06 & 0.002449 \\
\hline KEGG Pathway & hsa04020 & Calcium signaling pathway & $9.92 \mathrm{E}-06$ & 0.002449 \\
\hline KEGG Pathway & hsa04512 & ECM-receptor interaction & $3.29 \mathrm{E}-05$ & 0.004115 \\
\hline KEGG Pathway & hsa04142 & Lysosome & 8.43E-05 & 0.006504 \\
\hline KEGG Pathway & hsa04614 & Renin-angiotensin system & 0.000512 & 0.01489 \\
\hline KEGG Pathway & hsa05410 & Hypertrophic cardiomyopathy & 0.000845 & 0.01988 \\
\hline KEGG Pathway & hsa04974 & Protein digestion and absorption & 0.001509 & 0.030804 \\
\hline KEGG Pathway & hsa04392 & Hippo signaling pathway -multiple species & 0.001559 & 0.030804 \\
\hline KEGG Pathway & hsa04360 & Axon guidance & 0.002453 & 0.04179 \\
\hline KEGG Pathway & hsa05231 & Choline metabolism in cancer & 0.002919 & 0.048069 \\
\hline \multicolumn{5}{|c|}{$\begin{array}{l}\text { Note: Term: KEGG pathway name. Database: the KEGG database contains two sub-libraries, one is } \\
\text { KEGG PATHWAY and the other is KEGG DISEASE. ID: KEGG pathway ID. The uncorrected p-value is } \\
\text { provided in the column with the heading 'p-vale'; the smaller the p-value, the difference is statistically } \\
\text { more significant, and a p-value of less than } 0.05 \text { is considered to indicate significant enrichment. p- } \\
\text { adjust: The corrected p-value. }\end{array}$} \\
\hline
\end{tabular}

Table 3

Enrichment analysis of down-regulated pathways

\begin{tabular}{|c|c|c|c|c|}
\hline Database & ID & Term & p-value & p-adjust \\
\hline KEGG Pathway & hsa03008 & Ribosome biogenesis in eukaryotes & $9.9 \mathrm{E}-07$ & 0.0005 \\
\hline KEGG Pathway & hsa00270 & Cysteine and methionine metabolism & 7.4E-05 & 0.0103 \\
\hline KEGG Pathway & hsa05205 & Proteoglycans in cancer & 0.00097 & 0.0854 \\
\hline KEGG Pathway & hsa00670 & One carbon pool by folate & 0.00104 & 0.0854 \\
\hline KEGG Pathway & hsa00230 & Purine metabolism & 0.00258 & 0.1621 \\
\hline KEGG Pathway & hsa05169 & Epstein-Barr virus infection & 0.0027 & 0.1621 \\
\hline KEGG Pathway & hsa03010 & Ribosome & 0.00313 & 0.1621 \\
\hline \multicolumn{5}{|c|}{$\begin{array}{l}\text { Note: Term: KEGG pathway name. Database: the KEGG database contains two sub-libraries, one is } \\
\text { KEGG PATHWAY and the other is KEGG DISEASE. ID: KEGG pathway ID. The uncorrected p-value is } \\
\text { provided in the column with the heading 'p-vale'; the smaller the p-value, the difference is statistically } \\
\text { more significant, and a p-value of less than } 0.05 \text { is indicate significant enrichment. p-adjust: The } \\
\text { corrected p-value. }\end{array}$} \\
\hline
\end{tabular}


Figures

Fig. 1

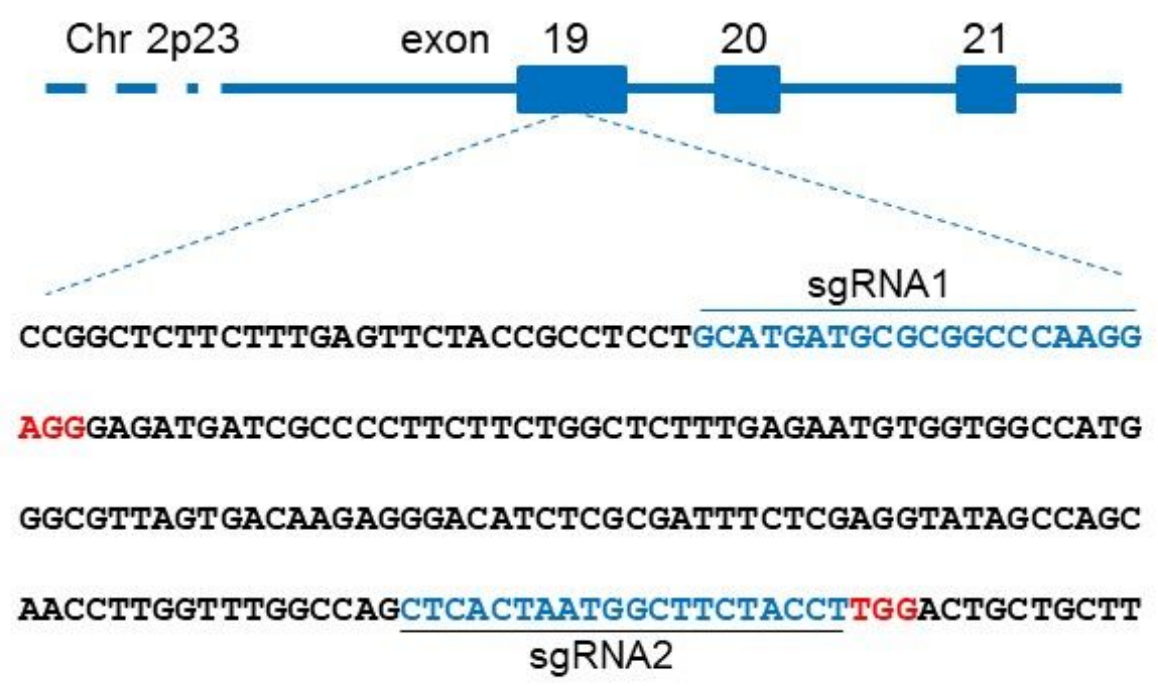

Figure 1

Location and design of sgRNAs. The sgRNA sequences are shown in blue, and protospacer adjacent motif (PAM) bases are in red. 
Fig. 2

a

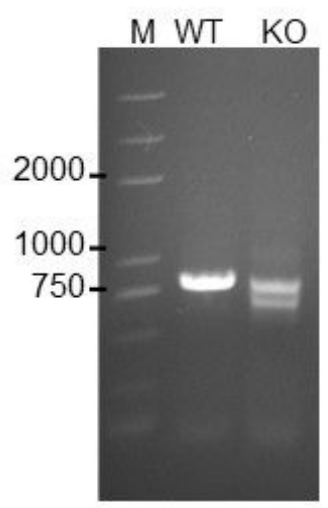

b

WT 5'-CCTGCATGATGCGCGGCCCAAGGAGG (114bp) CTCACTAATGGCTTCTACCTTGGACA-3'

KO A CCTGCATGATGCGCGGCCCA-_-CCTTGGACA $\triangle 137$ bp

KO в CCTGCATGATAGGAGG (114 bp) CTCACTAATGGCTTCTACCTTGGACA $\triangle 10 \mathrm{bp}$

\section{Figure 2}

Verification of DNMT3A knockout clone. a PCR identification of DNMT3A knockout. Lane M: Trans 2K DNA ladder. b Sanger sequencing results of PCR amplicons. KO A and KO B represent the two alleles of DNMT3A gene of DNMT3A KO cells. Blue bases: sgRNA sequences; Red bases: PAM. c Detection of DNMT3A protein expression with western blot. WT: wild type control, KO: DNMT3A KO cells. 
Fig. 3

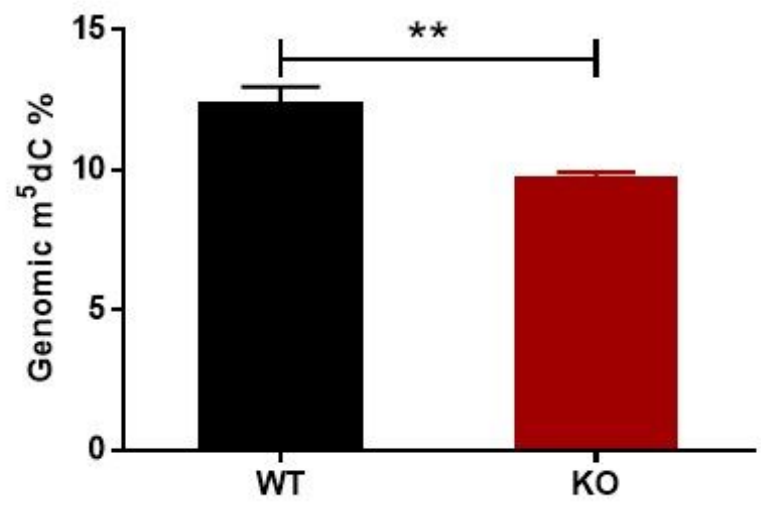

\section{Figure 3}

Comparison of methylation level of genomic DNA of wild type (WT) and DNMT3A knockout (KO) HEK293 cells. The methylation level of genomic DNA decreased by $21.5 \%$ due to DNMT3A deficiency. ${ }^{* *} p<0.01$ by two tailed students' t-test. 
Fig. 4

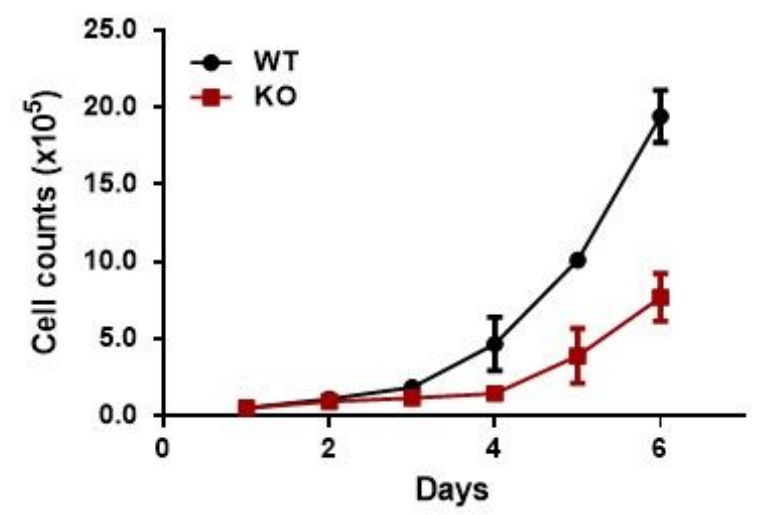

Figure 4

DNMT3A deficiency impaired cell growth. WT: wild type cells; KO: DNMT3A KO cells. 
Fig. 5

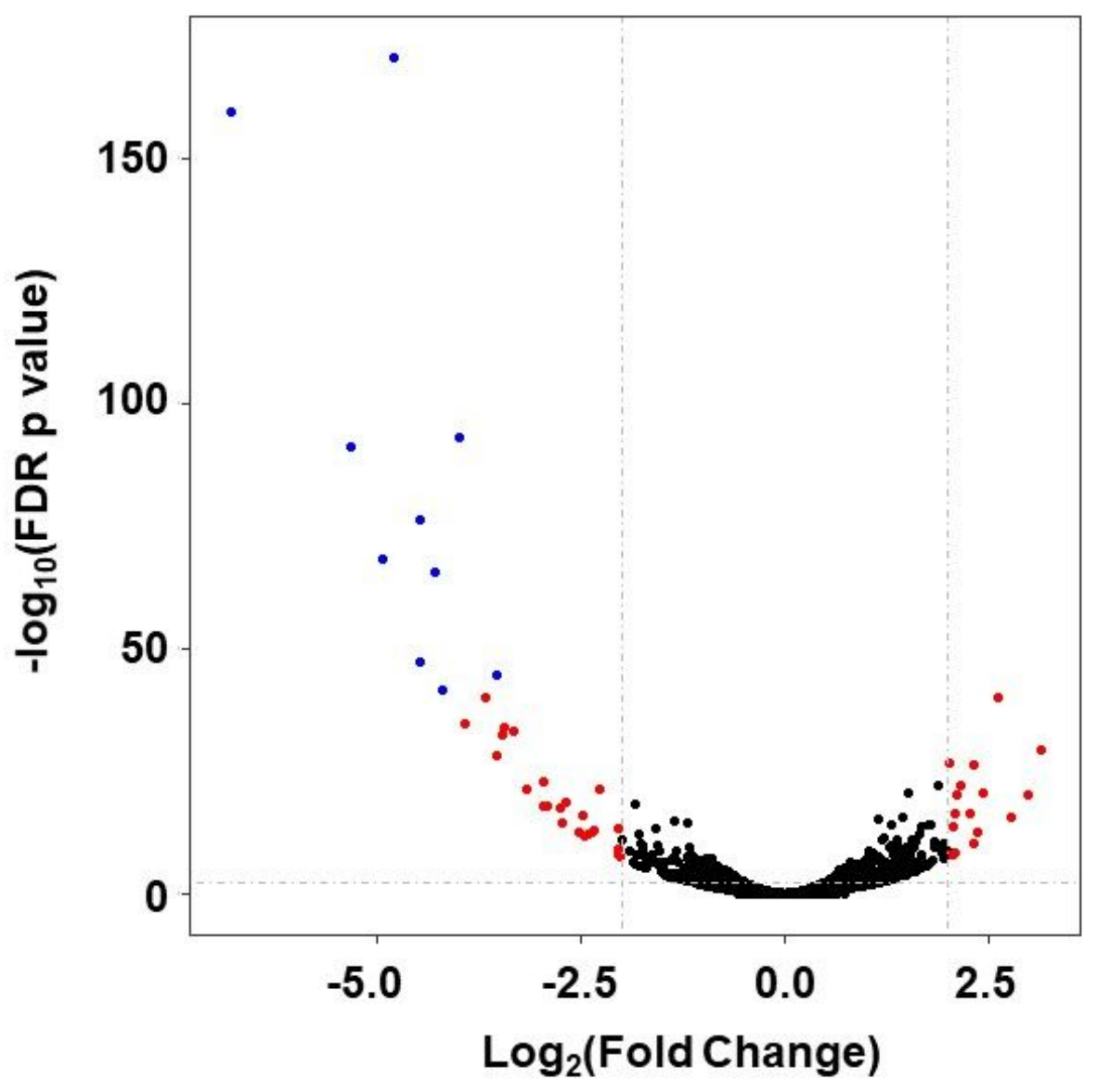

Figure 5

The significance and fold change of differential gene expression induced by DNMT3A deficiency. Each dot represents a gene; the significantly differentially expressed genes (FDR $p$ value $\leq 0.05$ and fold change $\geq 2$ ) are shown in red; the blue dots are top 10 differentially expressed genes (FDR p value $\leq 2.46 \times 10-42)$. 
Fig. 6

a

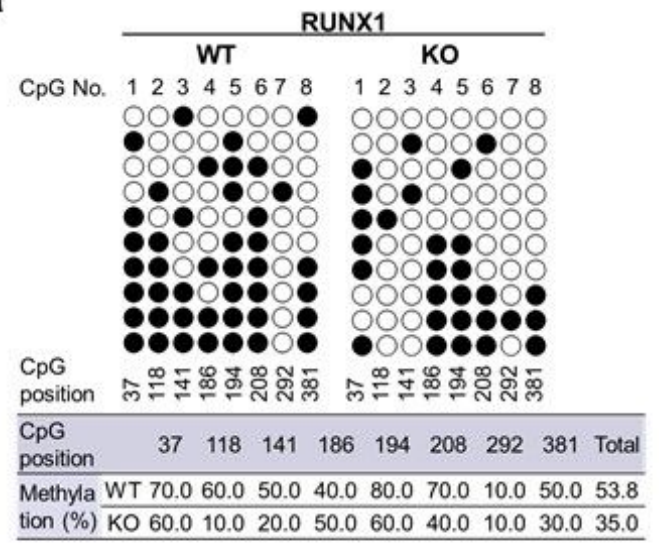

b

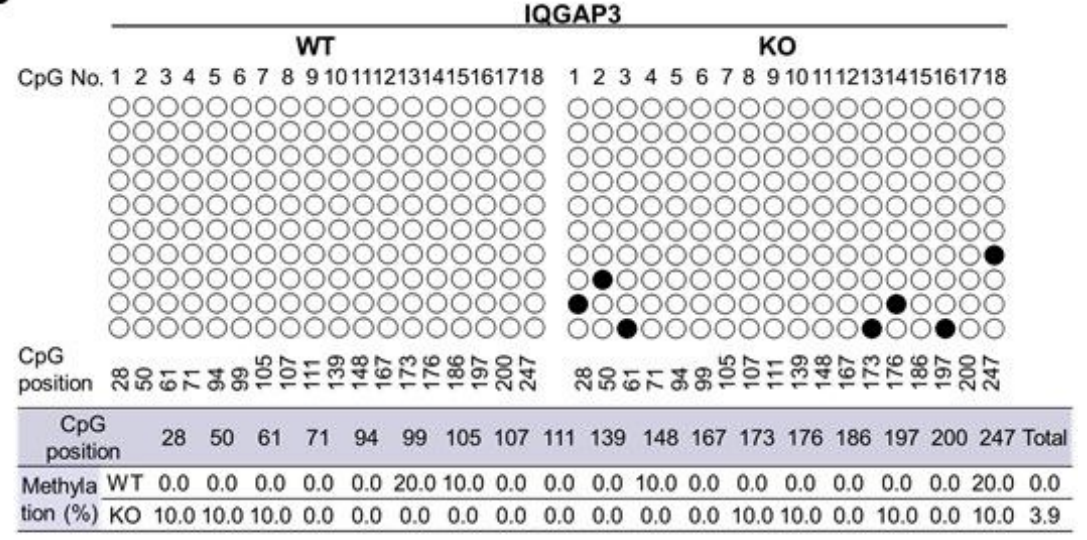

C

\begin{tabular}{l} 
DNMT3B \\
\hline WT KO
\end{tabular}

CPG No. $1233456789101112 \quad 123456789101112$

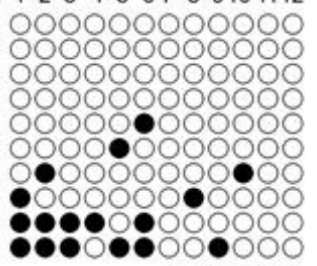

CpG

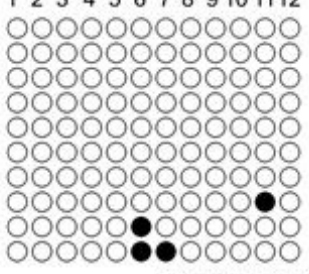

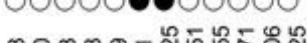

d

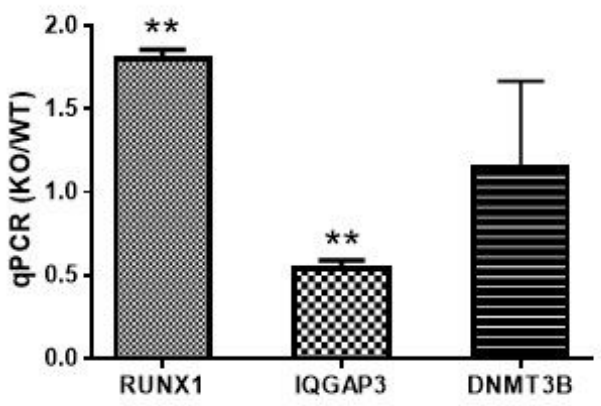

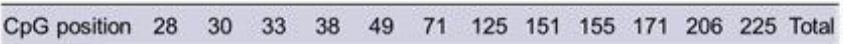
\begin{tabular}{llllllllllllll}
\hline Methyla WT 30.0 & 30.0 & 20.0 & 10.0 & 20.0 & 30.0 & 0.0 & 10.0 & 10.0 & 10.0 & 0.0 & 0.0 & 14.2 \\
\hline
\end{tabular}

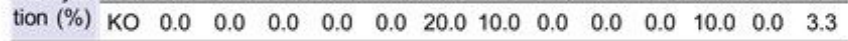

\section{Figure 6}

DNA methylation levels and quantitative PCR evaluation of the three representative genes, RUNX1, IQGAP3, and DNMT3B, regulated by DNMT3A deficiency. Bisulfite analysis showed that, in comparison with WT cells, the promoter methylation level of RUNX1 is decreased by DNMT3A deficiency (a), while the IGAPQ3 promoter has more methylated $\mathrm{CpG}$ islands in DNMT3A KO cells (b). DNMT3B promoter methylation is also regulated by DNMT3A and decreases upon its deficiency (c). d Quantitative PCR was performed in triplicate for mRNA expression profiles. In comparison with the relative transcription levels (to GAPDH level) in WT cells, RUNX1 transcription levels were significantly up-regulated by $80 \%$, IQGAP3 transcription levels were reduced by $46 \%$, and DNMT3B transcription levels were up-regulated by about $15 \%$. ${ }^{*} \mathrm{p}<0.01$ by two tailed students' t-test. 
Fig. 7

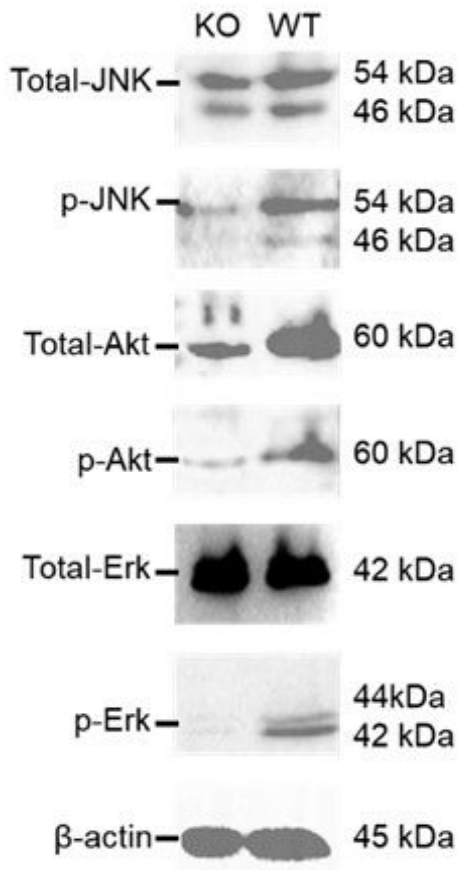

Figure 7

DNMT3A deficiency suppressed MAPK and PI3K-Akt pathways. Total proteins as well as phosphorylated fractions of Erk, JNK, and Akt were detected with $\beta$-actin as the housekeeping control.

\section{Supplementary Files}

This is a list of supplementary files associated with this preprint. Click to download.

- Supplementarydata20200725.docx 